Topol ogy opt i mizat i on of nonl i near opt i cal wavegui de devi ces consi dering out put si gnal phase

\begin{tabular}{|l|l|}
\hline 著者 & $\begin{array}{l}\text { MORI Koyo, MORI MOTO Kei ta, TANAKA Tonohi r o, } \\
\text { I GUCH Aki to, TSUJ I Yasuhi de }\end{array}$ \\
\hline $\begin{array}{l}\text { j our nal or } \\
\text { publ i cat i on t i t l e }\end{array}$ & Opt i cs Communi cat i ons \\
\hline vol une & 439 \\
\hline page range & $290-294$ \\
\hline year & 2019- 05- 15 \\
\hline URL & ht t p: //hdl . handl e. net /10258/00009956 \\
\hline
\end{tabular}




\title{
Topology optimization of nonlinear optical waveguide devices considering output signal phase
}

\author{
Koyo Mori, Keita Morimoto, Tomohiro Tanaka, Akito Iguchi, Yasuhide Tsuji* \\ The Division of Information and Electronic Engineering, Muroran Institute of Technology, Muroran, 050-8585 Japan
}

\begin{abstract}
A signal output of optical devices with multi-input ports, such as a logic gate, depends on the phase difference between input ports. In order to cascade optical devices, it is essential to design such devices considering not only desired output power but also desired output signal phase. We propose a topology optimization method for optical devices considering the output signal phase. In our approach, the beam propagation method (BPM) is employed as a numerical simulation method and the adjoint variable method (AVM) is used to calculate the sensitivity of output power and signal phase to design parameters. The validity and the effectiveness of our approach are verified through design examples of a three-branching waveguide with linear media and an all-optical logic gate utilizing nonlinear media.
\end{abstract}

Keywords: Topology optimization, beam propagation method (BPM), adjoint variable method(AVM), optical waveguide device, all-optical logic gate.

\section{Introduction}

In recent years, all-optical signal processing technology of optical communication systems have been expected to be established with the increase of communication traffic. In order to respond to these demands, nonlinear optical waveguide devices as ultrafast all-optical switching and logic devices have been attracted wide attention. Therefore, a lot of nonlinear waveguide devices utilizing the optical Kerr effect have been proposed [1-5].

Topology optimal design methods which can automatically generate a novel device structure without requiring any designer' s knowledge and experience attract a lot of attention [6-16]. Optimization methods have been proposed by combining a numerical simulation method and a search method so far. The finite element method (FEM) [6-11], the finite difference time domain (FDTD) method $[12,13]$, or the beam propagation method $(\mathrm{BPM})[14-$ 16] has been utilized as a numerical simulation method in these topology optimization method. Among them, BPM [17-19] is a quite useful technique in the analysis of long-length waveguide devices in which backward reflection hardly occurs. BPM can treat the large-scale problem which is difficult to be analyzed by FEM or FDTD due to the lack of computational resources. In the gradient-based optimal design methods, it is important to efficiently analyze the sensitivity which is the characteristic change depending on the change of each design parameter. For that reason, the adjoint variable method (AVM) for BPM has been proposed and has been also applied to the topology optimal design.

In the past topology optimization based on BPM and AVM [13], only linear materials are considered. To realize flexible photonic network, various functional optical devices are required and optical devices which can control light by light, such as the optical switch, logic gate, and so on are important optical components. In order to use these optical devices in cascade, it is important to control the signal phase because the transmission property of multi-input devices depends on the phase difference between input ports[5]. However, in the previous study, only the desired transmission power is considered and the output signal phase is not considered.

In this paper, we propose a design optimization method considering the output signal phase in which the sensitivity of phase is also evaluated by AVM. Furthermore, we also extend the topology optimization method based on BPM and AVM in order to design Kerr-type nonlinear optical waveguide devices.

In Section 2, we extend the formulation of the topology optimization utilizing BPM and AVM in order to design nonlinear optical devices. The sensitivity analysis of the output signal phase is also described. After that, the design examples of our approach are shown in Section 3 and the conclusion is given in Section 4.

\footnotetext{
* Corresponding author.

Email address: y-tsuji@mmm.muroran-it.ac.jp (Yasuhide Tsuji )
} 


\section{TOPOLOGY OPTIMIZATION FOR NONLIN- EAR DEVICES}

\subsection{Wave Equation}

We consider a 2-dimensional optical waveguide whose structure is uniform in the $x$ direction, as shown in Fig. 1, where $y$ and $z$ are the transverse and propagation directions, respectively. From Maxwell's equations, the wave equation for the lightwave in an optical waveguide is expressed as follows:

$$
\frac{\partial}{\partial y}\left(p \frac{\partial \Phi}{\partial y}\right)+\frac{\partial}{\partial z}\left(p \frac{\partial \Phi}{\partial z}\right)+k_{0}^{2} q \Phi=0,
$$

where $k_{0}$ is the free space wavenumber and $\Phi, p$, and $q$ are defined as follows:

$$
\begin{array}{llll}
\Phi=E_{x}, & p=1, & q=n^{2} & \text { for TE wave } \\
\Phi=H_{x}, & p=1 / n^{2}, & q=1 & \text { for TM wave. }
\end{array}
$$

By assuming the reference refractive index in BPM be $n_{\text {ref }}$, electromagnetic field, $\Phi$, is represented by slowly varying amplitude, $\phi$, and a steady-state propagation as follow:

$$
\Phi(y, z)=\phi(y, z) \exp \left(-j k_{0} n_{\text {ref }} z\right) .
$$

Substituting (2) into (1) and employing Fresnel approximation, the basic equation for beam propagation analysis is obtained as

$$
\begin{aligned}
-j 2 p k_{0} n_{\text {ref }} \frac{\partial \phi}{\partial z}+\frac{\partial}{\partial y}( & \left(p \frac{\partial \phi}{\partial y}\right) \\
& +k_{0}^{2}\left(q-p n_{\text {ref }}^{2}\right) \phi=0
\end{aligned}
$$

Then, applying the Crank-Nicolson scheme to the propagation direction of (3), the sequential updating equation can be expressed as follows[13]:

$$
\left[A\left(\phi_{j+1}\right)\right]_{j}\{\phi\}_{j+1}=\left[B\left(\phi_{j}\right)\right]_{j}\{\phi\}_{j},
$$

where $\left[A\left(\phi_{j+1}\right)\right]_{j},\left[B\left(\phi_{j}\right)\right]_{j}$ are matrix operators obtained at $j$-th propagation step. In the case that optical devices with nonlinear media are analyzed, these matrices depend on the electromagnetic field intensity. Considering Kerrtype nonlinear media, refractive index depends on the electric field intensity as follows[19]:

$$
\begin{aligned}
n & =n_{0}+\frac{1}{2} c n_{0} n_{2} \varepsilon_{0}|\boldsymbol{E}|^{2} \\
& =n_{0}+n_{2} I
\end{aligned}
$$

where $n_{0}$ is a linear refractive index, $n_{2}$ is a nonlinear refractive index, $c$ is a velocity of light, $\varepsilon_{0}$ is a permittivity in free-space, $I$ is an optical intensity.

It is known that, in a nonlinear waveguide analysis using the conventional BPM, the propagation step size $\Delta z$ has to be very small compared with the wavelength to ensure the computational accuracy[19]. That makes the computational time far longer compared with the case of linear waveguide devices. In the present topology optimization,

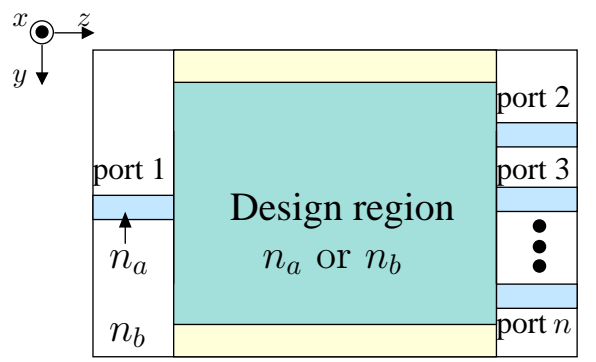

Figure 1: 2-dimensional optical waveguides

BPM analysis has to be iteratively performed until an optimized structure is obtained. For an efficient BPM analysis we employ BPM with an iterative scheme in the single propagation step[20]. In our previous discussion[20], it is shown that only three times of iterations are required in the single propagation step when using the same propagation step size with that in the linear waveguide analysis.

\subsection{Representation of Refractive Index Distribution}

We employ the density method to express the refractive index in the design region. In this case, the refractive index distribution is represented by the normalized density distribution $\rho_{i j}[13]$ where $i$ and $j$ denote discretized coordinate along $y$ and $z$ directions, respectively. Although $\rho_{i j}$ should be 0 or 1 in the actual device structure, in order to estimate the sensitivity of the transmission property to design parameters, those values may become intermediate values between 0 and 1 during the optimization. This corresponds to the intermediate material between core and cladding. In order to control this gray region, the refractive index distribution in the design region is represented as follows by using a modified Heaviside function:

$$
\begin{aligned}
n_{i j} & =n_{0, i j}+n_{2, i j} I, \\
n_{0, i j}^{2} & =n_{b 0}^{2}+\left(n_{a 0}^{2}-n_{b 0}^{2}\right) H\left(\rho_{i j}\right), \\
n_{2, i j} & =n_{b 2}+\left(n_{a 2}-n_{b 2}\right) H\left(\rho_{i j}\right), \\
H\left(\rho_{i j}\right) & =\left\{\begin{array}{ll}
\frac{1}{2}\left(2 \rho_{i j}\right)^{m} & \left(0 \leq \rho_{i j}<\frac{1}{2}\right) \\
1-\frac{1}{2}\left(2-2 \rho_{i j}\right)^{m} & \left(\frac{1}{2} \leq \rho_{i j}\right)
\end{array},\right.
\end{aligned}
$$

where $n_{a}$ and $n_{b}$ are the refractive indices of the core and cladding materials, respectively, and $m$ is a penalty parameter. If $m$ is a large value in the initial phase of optimization, the sensitivity is localized around the waveguide boundaries and the topology of the device structure may hardly changes. Therefore, in this paper, $m$ is linearly increased from 1 to 64 as the optimization proceeds. Finally, the remaining gray region is binarized by the threshold value of 0.5 .

\subsection{Sensitivity Analysis Based on Adjoint Variable Method}

We describe the sensitivity analysis when using the FD-BPM and the density method. We assume the objective function $C$ can be expressed by the normalized power 
$\left|S_{n 1}\right|^{2}$ and the signal phase $\theta$, where $S_{n 1}\left(=\left|S_{n 1}\right| e^{j \theta_{n 1}}\right)$ is an S-parameter from port 1 to port $n$. In the case of FDBPM analysis, $S_{n 1}$ is expressed as follows:

$$
\begin{aligned}
S_{n 1} & =\int \psi_{n}^{*} \phi_{N} d y=\int p \phi_{n}^{*} \phi_{N} d y \\
& =\left\{g_{n}\right\}^{T}\{\phi\}_{N},
\end{aligned}
$$

where, $\psi_{n}$ is the eigenmode field of port $n$ and $\left\{g_{n}\right\}$ is expressed as follows:

$$
\left\{g_{n}\right\}=\Delta y\left\{\psi_{n}^{*}\right\}=p \Delta y\left\{\phi_{n}^{*}\right\},
$$

where $\Delta y$ is the step size in the $y$ direction. Using sequential updating equation (4), the output field $\{\phi\}_{N}$ can be calculated by

$$
\{\phi\}_{N}=\left\{\prod_{j=0}^{N-1}\left([A]_{j}^{-1}[B]_{j}\right)\right\}\{\phi\}_{0},
$$

$S_{n 1}$ is expressed as

$$
S_{n 1}=\left\{g_{n}\right\}^{T}[A]_{N-1}^{-1}[B]_{N-1} \cdots[B]_{0}\{\phi\}_{0} .
$$

Since the matrices $[A]_{j}$ and $[B]_{j}$ depend on $\rho_{i j}$, by differentiating (13) with respect to $\rho_{i j}$, we can get the sensitivity of $S_{n 1}$ as follows[13]:

$$
\begin{aligned}
\frac{\partial S_{n 1}}{\partial \rho_{i j}} & =\frac{\partial\left\{\lambda_{n}\right\}_{j}^{T}}{\partial \rho_{i j}}[B]_{j}\{\phi\}_{j}+\left\{\lambda_{n}\right\}_{j}^{T} \frac{\partial[B]_{j}}{\partial \rho_{i j}}\{\phi\}_{j} \\
& =\left\{\lambda_{n}\right\}_{j}^{T}\left(-\frac{\partial[A]_{j}}{\partial \rho_{i j}}\{\phi\}_{j+1}+\frac{\partial[B]_{j}}{\partial \rho_{i j}}\{\phi\}_{j}\right),
\end{aligned}
$$

with

$$
\left\{\lambda_{n}\right\}_{j}^{T}=\left\{g_{n}\right\}^{T}[A]_{N-1}^{-1}[B]_{N-1} \cdots[B]_{j+1}[A]_{j}^{-1} .
$$

From (14), we can see that the sensitivities to every design parameters are estimated by only a forward and backward propagation analysis. In the calculation of $\left\{\lambda_{n}\right\}_{j}$, how to treat the refractive index change caused by nonlinear effect is an important issue. In this paper, we assume that the nonlinear effect occurs by the electric field obtained in the forward propagation analysis even in the backward propagation analysis.

Once we can get the sensitivity of $S_{n 1}$, the sensitivity of power and phase can be easily calculated because $S_{n 1}$ has information of both power and phase. The sensitivity of the transmission power, $\left|S_{n 1}\right|^{2}$, is expressed as follows:

$$
\frac{\partial\left|S_{n 1}\right|^{2}}{\partial \rho_{i j}}=\operatorname{Re}\left\{2 S_{n 1}^{*} \frac{\partial S_{n 1}}{\partial \rho_{i j}}\right\} .
$$

The output signal phase, $\theta_{n 1}$, is expressed as

$$
\theta_{n 1}=\tan ^{-1} \frac{\operatorname{Im}\left\{S_{n 1}\right\}}{\operatorname{Re}\left\{S_{n 1}\right\}},
$$

and its sensitivity is expressed as follows:

$$
\begin{aligned}
\frac{\partial \theta_{n 1}}{\partial \rho_{i j}} & =\frac{1}{1+\left(\frac{\operatorname{Im}\left\{S_{n 1}\right\}}{\operatorname{Re}\left\{S_{n 1}\right\}}\right)^{2}} \cdot \frac{\operatorname{Im}\left\{\frac{\partial S_{n 1}}{\partial \rho_{i j}} S_{n 1}^{*}\right\}}{\left(\operatorname{Re}\left\{S_{n 1}\right\}\right)^{2}} \\
& =\operatorname{Im}\left\{\frac{\partial S_{n 1}}{\partial \rho_{i j}} \cdot \frac{1}{S_{n 1}}\right\} .
\end{aligned}
$$

\subsection{Update of Refractive Index Distribution}

In this study, we employ a gradient descent method to update design parameters. The design parameters are updated by following equation:

$$
\begin{aligned}
\boldsymbol{\rho}^{\text {post }}=\boldsymbol{\rho}^{\text {pre }} & +\alpha \times\left(-\nabla_{\rho} C_{\text {power }}\right) \\
& +\beta \times\left(-\nabla_{\rho} C_{\text {phase }}\right),
\end{aligned}
$$

where $\boldsymbol{\rho}^{\text {post }}$ and $\boldsymbol{\rho}^{\text {pre }}$ are the vector consist of post-update and pre-update density parameters, respectively. $\nabla_{\rho}$ is the gradient operator with respect to density parameters, and $C_{\text {power }}$ and $C_{\text {phase }}$ are the objective functions to be minimized for the normalized power and signal phase, respectively. The step size to update design parameters is decided by proportional constant $\alpha$ and $\beta$. In this paper, those values are taken to be as follows:

$$
\begin{aligned}
& \alpha=\frac{C_{\text {opt }, \text { power }}-C_{\text {power }}}{\left\|\nabla_{\rho} C_{\text {power }}\right\|} \delta_{1}, \\
& \beta=\frac{C_{\text {opt,phase }}-C_{\text {phase }}}{\left\|\nabla_{\rho} C_{\text {phase }}\right\|} \delta_{2} \times\left(1-\left|C_{\text {opt } \text { power }}-C_{\text {power }}\right|\right),
\end{aligned}
$$

where $C_{\text {opt,power }}$ and $C_{\text {opt,phase }}$ are the optimum values for the objective function of the normalized power and output signal phase, respectively. $\left\|\nabla_{\rho} C_{\text {power }}\right\|$ and $\left\|\nabla_{\rho} C_{\text {phase }}\right\|$ are the vector norms of $\nabla_{\rho} C_{\text {power }}$ and $\nabla_{\rho} C_{\text {phase }}$, respectively. $\delta_{1}$ and $\delta_{2}$ are constant values which are appropriately selected depending on design problems.

\subsection{Weighted Moving Average Filter For Structural Smooth- ing}

Since the topology optimization has large design freedom, emergence of fine structures, typified by a checkerboard pattern, has been reported and should be suppressed. We employ structural smoothing filter which can remove such fine structures. In this paper, using a weighted moving averaging filter, density parameter distribution $\rho_{i j}$ is filtered as follows:

$$
\begin{gathered}
\tilde{\rho}_{i j}=\sum_{m=-1}^{1} \sum_{n=-1}^{1} w_{m n} \rho_{(i+m)(j+n)}, \\
w_{m n}=\frac{1}{W+8} \times\left\{\begin{array}{ll}
W & (m=n=0) \\
1 & (m \neq 0 \text { or } n \neq 0)
\end{array},\right.
\end{gathered}
$$

where $W$ is weight coefficient. If smoothing effect is strong, transmission characteristics are not possibly improved. Thus, in this paper, we select $W$ to be 5 . 


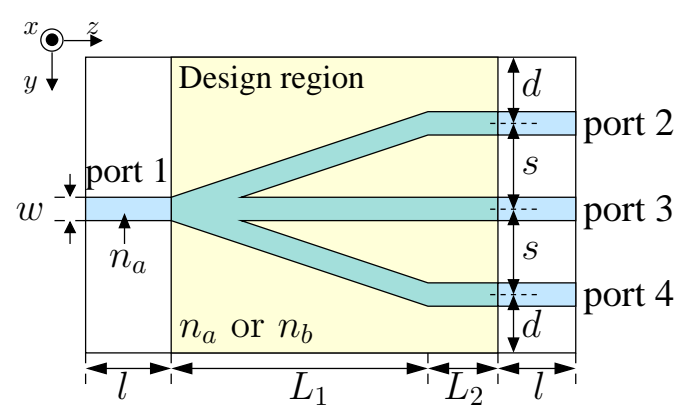

Figure 2: Design model of a three-branching waveguide.

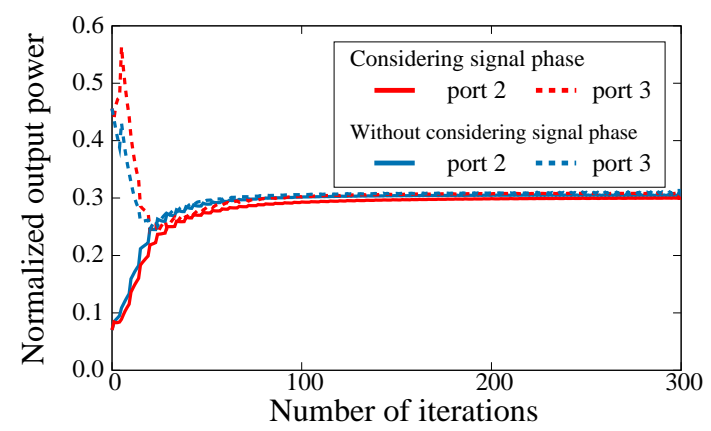

(a) Normalized output power.

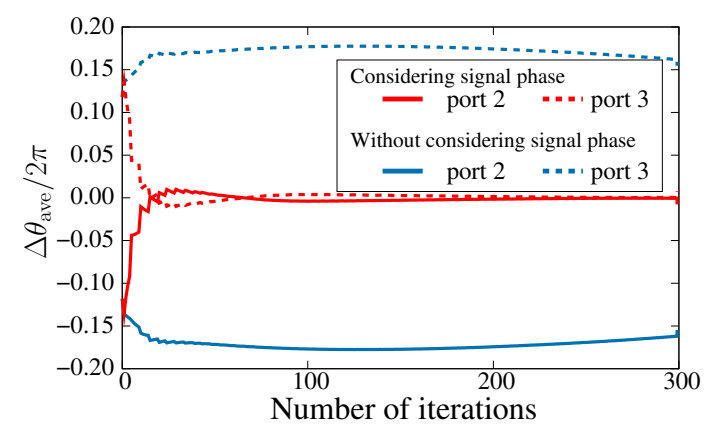

(b) Output signal phase.

Figure 3: Output characteristics during the optimization process in the design of a power divider.

\section{Design examples}

\subsection{Power Divider With Equal Phase}

In order to confirm the validity of our approach for optimization to get desired output power and signal phase, first, we consider an equal power divider with equal output phase. The design model of this power divider is shown in Fig.2. In this design example, we consider the linear dielectric material and the refractive indices of the core and cladding are assumed to be $n_{1}=1.45$ and $n_{2}=1.445$, respectively. The structural parameters are as follows: $L_{1}=400 \mu \mathrm{m}, L_{2}=50 \mu \mathrm{m}, l=50 \mu \mathrm{m}, w=5.0 \mu \mathrm{m}$, $s=18 \mu \mathrm{m}, d=22 \mu \mathrm{m}$. We assume a fundamental TE mode at the wavelength of $1.55 \mu \mathrm{m}$ is launched into port 1. In this optimal design, three-branching waveguide is given as an initial structure. The objective function to be

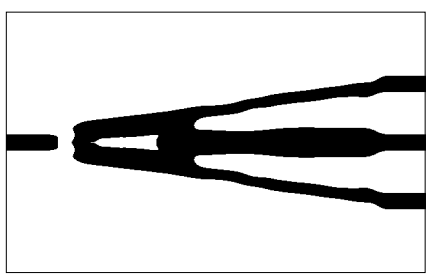

(a)

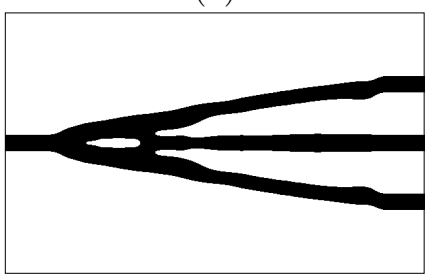

(c)

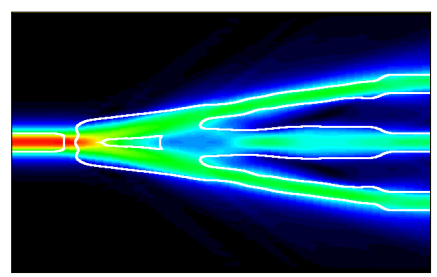

(b)

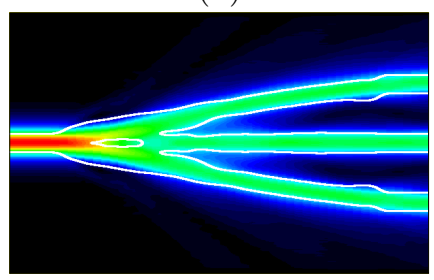

(d)
Figure 4: Optimized structure and propagation wave in the design of a power divider. (a) and (b): considering output phase, (c) and (d): without considering output phase

minimized is given as follows:

$$
\begin{aligned}
\text { Minimize } C & =C_{\text {power }}, C_{\text {phase }} \\
C_{\text {power }} & =\sum_{n=2}^{4}\left(\frac{1}{3}-\left|S_{n 1}\right|^{2}\right)^{2}, \\
C_{\text {phase }} & =\sum_{n=2}^{4}\left(\theta_{\text {ave }}-\theta_{n 1}\right)^{2},
\end{aligned}
$$

where $\theta_{\text {ave }}$ is the average phase of the output signals at previous iteration step during optimization, $\delta_{1}$ and $\delta_{2}$ are set to be 8.0 and 3.0, respectively. The step sizes are $\Delta y=0.2 \mu \mathrm{m}$ and $\Delta z=1 \mu \mathrm{m}$. We impose a symmetry condition with respect to the $y$-direction considering the desired device characteristics. In addition, we apply the smoothing filter every 20 iterations. The normalized output power and signal phase are shown in Fig.3 as a function of the number of iterations. For a comparison with the conventional design approach[13], the results without considering signal phase are also shown in the same figure. In both case with and without considering signal phase, we can see that the normalized output power is monotonically improved with an iteration. On the other hand, the phase difference is able to be suppressed in the present approach. The optimized structures and propagation fields in both cases are shown in Fig.4. The normalized output power into port 2 and port 3 respectively are 0.297 and 0.309 in Figs.4(a), (b), and 0.318 and 0.302 in Figs.4(c), (d). Normalized phase difference is $\Delta \theta / 2 \pi=15.44 \times 10^{-3}$ in Figs.4(a), (b), $\Delta \theta / 2 \pi=30.93 \times 10^{-2}$ in Figs.4(c), (d). From the optimized structure, we can observe that phase difference is suppressed by adjusting width of waveguide. 


\subsection{All Optical NAND Gate}

Next, we consider a design example of an all-optical NAND gate using Kerr nonlinearity. Since logic gates are generally used in cascaded form, the output signal phase has to be controlled for the next stage operation. The design model of the NAND gate is shown in Fig.5. The material parameters are assumed to be $n_{a}=n_{a 0}+n_{a 2} I$, $n_{a 0}=1.57, n_{a 2}=1.0 \times 10^{-9} \mathrm{~m}^{2} / \mathrm{W}($ for MBBA Liquid Crystal)[21], and $n_{b}=1.55$. The structural parameters are as follows: $L=300 \mu \mathrm{m}, l=50 \mu \mathrm{m}, w=2.5 \mu \mathrm{m}$, $s=8 \mu \mathrm{m}, d=17 \mu \mathrm{m}$. A fundamental TE mode at the wavelength of $1.3 \mu \mathrm{m}$ is launched and the input power is set to be $P_{\text {in }}=15 \mathrm{~W} / \mathrm{m}$. A truth table of the desired NAND gate is shown in Table I. Each power is normalized by $P_{\text {in }} . P_{i}(i=\mathrm{A}, \mathrm{B}, \mathrm{Ref})$ denotes the normalized power of each input port and $P_{o}(o=\mathrm{NAND}, \operatorname{Rad} 1, \operatorname{Rad} 2)$ denotes the normalized power of each output port. Port A and B are used as signal ports, and port Ref is used to realize truth state when no signal power is input in the logic00 state. Port Rad1 and Rad2 are used to escape unnecessary power. To realize the desired truth table shown in Table I, the objective function is set as follows:

$$
\begin{aligned}
& \text { Minimize } C=C_{\text {power }}, C_{\text {phase }} \\
& C_{\text {power }}=C_{00}+C_{10}+C_{11} \\
& C_{10}=2 \times\left(P_{\mathrm{NAND}, 10}^{\mathrm{opt}}-P_{\mathrm{NAND}, 10}\right)^{2} \\
& +\left(1-P_{\operatorname{Rad} 1,10}-P_{\operatorname{Rad} 2,10}\right)^{2}, \\
& C_{00}=W \sum_{X}\left(P_{X, 00}^{\mathrm{opt}}-P_{X, 00}\right)^{2}, \\
& C_{11}=W \sum_{X}\left(P_{X, 11}^{\mathrm{opt}}-P_{X, 11}\right)^{2}, \\
& (X=\text { NAND, } \operatorname{Rad} 1, \operatorname{Rad} 2) \\
& W= \begin{cases}2 & (X=\mathrm{NAND}) \\
1 & (X \neq \mathrm{NAND})\end{cases} \\
& C_{\text {phase }}=\left(\theta_{\text {ave }}-\theta_{\text {logic00 }}\right)^{2} \\
& +\left(\theta_{\text {ave }}-\theta_{\text {logic10 }}\right)^{2},
\end{aligned}
$$

where $C_{l}(l=00,10,11)$ is objective function of logicl and $P_{X, \mathrm{AB}}$ is the normalized output power into port $X$ when a signal corresponding to logicAB is given. $\theta_{\text {ave }}$ is the average phase of the output signals between logic00 and logic10 at previous iteration step during optimization. The step sizes are $\Delta y=0.1 \mu \mathrm{m}$ and $\Delta z=0.4 \mu \mathrm{m}$. The structure is assumed to be symmetric along the $y$-direction. Thus, logic01 is automatically satisfied when logic10 is satisfied. $\delta_{1}$ and $\delta_{2}$ are set to be 2.0 and 0.5 , respectively, and the smoothing filter is applied every 10 iteratons of optimization. The normalized output power and signal phase as a function of the number of iterations are shown in Fig.6. We can see that the normalized output power is improved with an iteration. We can also see that output signal phase difference is suppressed. Both characteristics of output power and signal phase are oscillatingly improved in the optimization process, these are suddenly fluctuated at about

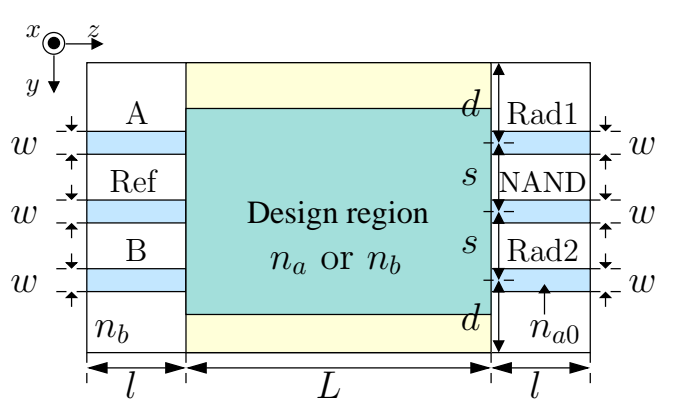

Figure 5: Design model of a optical NAND gate.

Table 1: True table of NAND gate.

\begin{tabular}{|c|c|c|c|c|c|}
\hline \multicolumn{3}{|c|}{ Input power } & \multicolumn{3}{c|}{ Output power } \\
\hline$P_{\text {Ref }}$ & $P_{\mathrm{A}}$ & $P_{\mathrm{B}}$ & $P_{\mathrm{NAND}}^{\mathrm{Opt}}$ & $P_{\mathrm{Rad} 1}^{\mathrm{opt}}$ & $P_{\text {Rad2 }}^{\text {opt }}$ \\
\hline 1 & 0 & 0 & 1 & 0 & 0 \\
\hline 1 & 0 & 1 & 1 & $X$ & $Y$ \\
\hline 1 & 1 & 0 & 1 & $Y$ & $X$ \\
\hline 1 & 1 & 1 & 0 & 1.5 & 1.5 \\
\hline
\end{tabular}

1100 iterations. This is caused by trying to simultaneously improve multi-objectives. In the design of logic gate, the objective function is expressed by the sum of the objectives for each logic state, and the terms for suppressing phase differences are also included in the objective function. To improve one objective may degrade the other objectives. As a result, each objective is not monotonically improve but oscillatingly improved.

The optimized structure and propagating field in each logic state are shown in Fig.7. On the NAND port, the normalized output power of better than 0.89 and extinction ratio of better than $-13.8 \mathrm{~dB}$ are obtained. In addition, the phase difference between logic00 and logic10 is as small as $\Delta \theta / 2 \pi=15.66 \times 10^{-3}$. By setting the threshold to be $35 \%$, we confirm that cascading up to 3 stages is available using this NAND gate. The limitation of the number of cascades mainly depends on the power reduction at the output port of NAND gate compared with each logic input. In this result, the normalized input power of the next stage becomes lower than 1 . In order to improve this situation and overcome the limitation of the number of cascades, we think that it is effective to design a NAND gate so that normalized output power in high state becomes greater than 1 by setting $P_{\text {Ref }}$ to be greater than 1. In this case, the extra power of NAND output should be attenuated by using an optical limiter[22] for adjusting the normalized input power of the next stage to be 1 . The optimal design for NAND gate combined with an optical limiter will be discussed in the future work.

\section{Conclusion}

In this paper, we proposed a design optimization considering the output signal phase. Moreover, we extended 


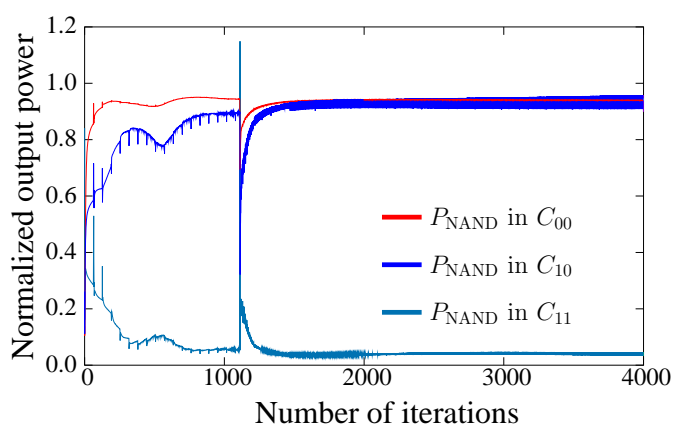

(a) Normalized output power.

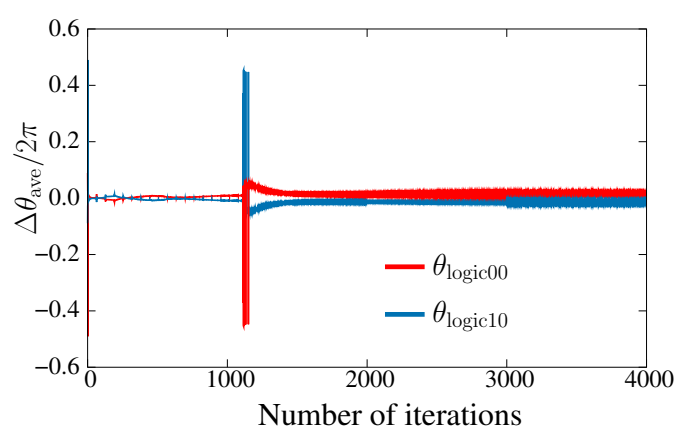

(b) Output signal phase.

Figure 6: Output characteristics during the optimization process in the design of a optical NAND gate.

the topology optimization method based on BPM and AVM to be able to design Kerr-type nonlinear optical waveguide devices. As design examples, a three-branching waveguide with linear media and an all-optical logic gate utilizing nonlinear media are designed to show the validity and usefulness of our approach. In the case of optical devices utilizing Kerr-type nonlinear media, propagation characteristics depend on an input power. Therefore, the design of nonlinear optical devices with high tolerance to incident power fluctuation is important and that is our future work.

\section{Acknowledgement}

This work was supported by JSPS KAKENHI Grant Number 18K04276.

\section{References}

[1] Y. D. Wu, and T. T. Shih and M. H. Chen, New all-optical logic gates based on the local nonlinear Mach-Zehnder interferometer, Opt. Express 16 (1) (2008) 248-257.

[2] Z. Mohebbi, N. Nozhat, and F. Emami, High contrast all-optical logic gates based on 2D nonlinear photonic crystal, Opt. Commun. 355 (15) (2015) 130-136.

[3] Y. Fu, X. Hu, and Q. Gong, Silicon photonic crystal all-optical logic gates, Phys. Lett. A 377 (3-4) 329-333.

[4] Y. D. Wu and M. L. Whang and M. H. Chen and R. Z. Tasy, All-optical switch based on the local nonlinear Mach-Zehnder interferometer, Opt. Express 15 (16) (2007) 9883-9892.

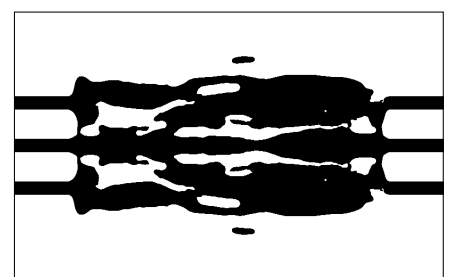

(a)

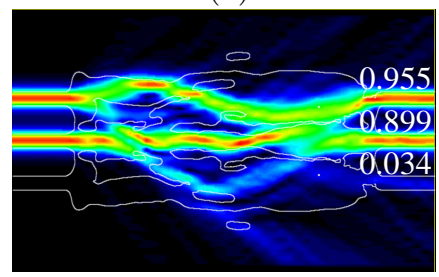

(c)

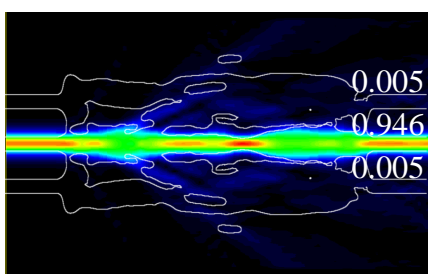

(b)

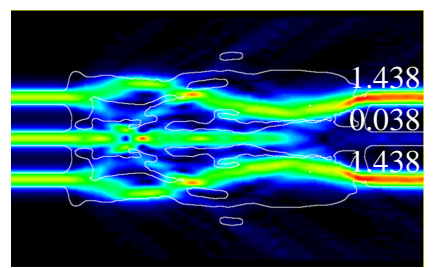

(d)
Figure 7: Optimized strcture and propagation wave in the design of a optical NAND gate. (a) Optimized structure. (b) logic00. (c) logic10. (d) logic11.

[5] V. Jandieri, R. Khomeriki and D. Erni, Realization of true all-optical AND logic gate based on nonlinear coupled air-hole type photonic crystal waveguides, Opt. Express 26 (16) (2018) 19845-19853.

[6] J. S. Jensen and O. Sigmund, Systematic design of photonic crystal structures using topology optimization: Low-loss waveguide bends, Appl. Phys. Lett. 84 (12) (2004) 2022-2024.

[7] Y. Tsuji, K. Hirayama, T. Nomura, K. Sato, and S. Nishiwaki , Design of optical circuit devices based on topology optimization, IEEE Photon. Technol. Lett. 18 (7) (2006) 850-852.

[8] Y. Tsuji and K. Hirayama, Design of optical circuit devices using topology optimization method with function-expansionbased refractive index distribution, IEEE Photon. Technol. Lett. 20 (12) (2008) 982-984.

[9] T. Yasui, Y. Tsuji, J. Sugisaka, and K. Hirayama, Design of three-dimensional optical circuit devices by using topology optimization method with function-expansion-based refractive index distribution, J. Lightw. Technol. 31 (23) (2013) 3765-3770.

[10] Z. Zhang, Y . Tsuji, T . Yasui, and K . Hirayama, Design of ultra-compact triplexer with function-expansion based topology optimization, Opt. Express 23 (4) (2015) 3936-3950.

[11] A. Tetu, M. Kristensen, L. H. Frandsen, A. Harpth, P. I. Borel, J. S. Jensen, and O. Sigmund, Broadband topology-optimized photonic crystal components for both TE and TM polarizations, Opt. Express 13 (21) (2005) 8606-8611.

[12] L. H. Frandsen, Y. Elesin, L. F. Frellsen, M. Mitrovic, Y. Ding, O. Sigmund, and K. Yvind, Topology optimized mode conversion in a photonic crystal waveguide fabricated in silicon-oninsulator material, Opt. Express 22 (7) (2014) 8525-8532.

[13] A . Iguchi , Y . Tsuji , T . Yasui, and K . Hirayama, Topology optimization of optical waveguide devices based on beam propagation method with sensitivity analysis, J. Lightw. Technol. 34 (18) (2016) 4214-4220.

[14] A . Iguchi , Y . Tsuji , T . Yasui, and K . Hirayama, Efficient topology optimization of optical waveguide devices utilizing semi-vectorial finite-difference beam propagation method, Opt. Express 25 (23) (2017) 28210-28222.

[15] A . Iguchi , Y . Tsuji, T . Yasui, and K . Hirayama, Topology optimal design for optical waveguides using time domain beam propagation method, IEICE Electron. Express 15 (2018) 20180417.

[16] Y. Chung and N. Dagli, An assessment of finite difference beam propagation method, IEEE Quantum Electron. 26 (8) (1990) 
$1335-1339$.

[17] Y. Tsuji and M. Koshiba, A finite element beam propagation method for strongly guiding and longitudinally varying optical waveguides, J. Lightw. Technol. 14 (2) (1996) 217-222.

[18] J. Yamauchi, S. Harada, S. Kobori, and H. Nakano, Semivector finite-difference formula for the analysis of a step-index waveguide with a tilted interface, IEEE Photon. Technol. Lett. 21 (24) (2009) 1867-1869.

[19] T . Yasui, M . Koshiba, and Y . Tsuji, A wide-angle finite element beam propagation method with perfectly matched layers for nonlinear optical waveguides, J. Lightw. Technol. 17 (10) (1999) 1909-1915.

[20] K. Mori and Y. Tsuji, A study on topology optimization of nonlinear optical device using beam propagation method and sensitivity analysis based on adjoint variable method, IEICE Trans. Electron. J101-C (5) (2018) 245-252.

[21] Y. D. Wu, Method for analyzing multilayer nonlinear optical waveguide, Opt. Express , 13 (20) (2005) 7982-7996.

[22] W. Wang, Y. Wang, K. Allaart, D. Lenstra, All-optical limiting using nonlinear directional couplers composed of a self-focusing and a self-defocusing waveguide, Appl. Phys. B , 83 (4) (2006) $623-628$. 